\title{
Sclareol Induces Plant Resistance to Root-Knot Nematode Partially Through Ethylene-Dependent Enhancement of Lignin Accumulation
}

\author{
Taketo Fujimoto, ${ }^{1,2,3}$ Takayuki Mizukubo, ${ }^{1}$ Hiroshi Abe, ${ }^{4}$ and Shigemi Seo ${ }^{2}$ \\ ${ }^{1}$ National Agricultural Research Center, Tsukuba, Ibaraki 305-8666, Japan; ${ }^{2}$ Plant-Microbe Interactions Research Unit, \\ National Institute of Agrobiological Sciences, Tsukuba, Ibaraki 305-8602, Japan; ${ }^{3}$ Research Fellow of Japan Society for the \\ Promotion of Science; ${ }^{4}$ Department of Biological Systems, RIKEN BioResource Center, Tsukuba, Ibaraki 305-0074, Japan
}

Submitted 17 February 2014. Accepted 11 November 2014.

\begin{abstract}
The root-knot nematode $(\mathrm{RKN})$ is one of the most devastating parasitic nematodes of plants. Although some secondary metabolites released by the host plant play roles as defense substances against parasitic nematodes, the mechanism underlying the induction of such defense responses is not fully understood. We found that sclareol, a natural diterpene known as an antimicrobial and defense-related molecule, inhibited RKN penetration of tomato and Arabidopsis roots. Sclareol induced genes related to ethylene (ET) biosynthesis and signaling and phenylpropanoid metabolism in Arabidopsis roots. In roots of $e$ in2-1, an ET-insensitive mutant line, both sclareolinduced inhibition of RKN penetration and sclareol-induced enhancement of lignin accumulation were abolished. A mutant defective in lignin accumulation did not exhibit such inhibition. Sclareol also activated MPK3 and MPK6, Arabidopsis mitogenactivated protein kinases whose activation is required for triggering ET biosynthesis. Sclareol-induced inhibition of RKN penetration was exhibited by mutants of neither MPK3 nor MPK6. Treatment with a biosynthetic precursor of ET was insufficient compared with sclareol treatment to inhibit RKN penetration, suggesting the existence of an ET-independent signaling pathway leading to $R K N$ resistance. These results suggested that sclareol induced resistance to RKN penetration partially through ET-dependent accumulation of lignin in roots.
\end{abstract}

Plants have evolved in complex environments in which they interact with many organisms. In order to survive in these environments, plants must overcome attack by harmful parasitic and phytophagous organisms such as bacteria, fungi, nematodes, and insects. Plants have developed defense systems with constitutive and inducible protective responses using different defense mechanisms against attack by specific types of organisms. The phytohormones jasmonic acid (JA), ethylene (ET), and salicylic acid (SA) play important roles in all of these responses (Robert-Seilaniantz et al. 2011). These plant hormones regulate various plant signal pathways, and early transcriptional effects have been linked to hormone response (Kazan and Lyons 2014). One of the transcriptional reactions in defense response includes the accumulation and

Corresponding authors: S. Seo; E-mail: sseo71@ affrc.go.jp; and T. Mizukubo; E-mail: mizu@ affrc.go.jp

* The $e$-Xtra logo stands for "electronic extra" and indicates that five supplementary figures and one supplementary table are published online.

(c) 2015 The American Phytopathological Society release of metabolites, known as secondary metabolites. Secondary metabolites such as isoprenoids, phenylpropanoids, alkaloids, and fatty acids or polyketides play an important role in both constitutive and inducible direct defense of plants against their natural enemies (Cheng et al. 2007; Vogt 2010; Ziegler and Facchini 2008).

The isoprenoid pathway produces terpenes, which are composed of five carbon-isoprene units. These are classified as mono, sesqui-, di-, tri, and higher-terpenes based on the number of isoprene units. Terpenes play roles in various physiological processes such as growth, development, responses to stresses, and disease resistance in plants (Cheng et al. 2007). Among the terpenes, the diterpene sclareol is of particular interest because of its range of physiological activities. Sclareol, which was originally isolated as a constituent of the essential oil in clary sage (Salvia sclarea), has been reported to be found in only a few plant species, including Nicotiana spp. (Caniard et al. 2012). Earlier studies showed that sclareol possessed direct antimicrobial activity against some types of plant-pathogenic bacteria and fungi (Bailey et al. 1975; Kennedy et al. 1992). Sclareol was demonstrated to be transported by $\mathrm{NpABC1}$, a stress-responsive ATPbinding cassette (ABC) transporter, in Nicotiana plumbaginifolia (Jasiński et al. 2001). Treatment of Nicotiana spp. plants with sclareol induced the expression of various genes, such as those encoding proteins involved in metabolism, disease resistance, transport, and hormone biosynthesis and signaling (Campbell et al. 2003; Grec et al. 2003; Jasiński et al. 2001; Seo et al. 2012). Such induction of defense-related genes by exogenously applied sclareol was also observed in Arabidopsis, a plant species that does not produce sclareol (Campbell et al. 2003). This suggested that there are certain signaling pathways activated by sclareol that are common in plants. Furthermore, sclareol conferred resistance to the soilborne bacterial pathogen Ralstonia solanacearum in tobacco, tomato, and Arabidopsis; and transgenic tobacco with reduced expression of $N t P D R 1$, a tobacco homolog of $N p A B C 1$, exhibited attenuated sclareol-induced resistance (Seo et al. 2012). These findings suggested that sclareol is involved in defense responses to biotic stresses in plants.

Plant-parasitic nematodes parasitize roots or stems of various plants, thereby inhibiting absorption of nutrients and moisture (Wyss et al. 1992). Root-knot nematodes (RKNs) (Meloidogyne spp.) deserve careful attention because they have a wide host range, and RKN parasitization causes severe crop damage. RKNs are sedentary endoparasitic nematodes. Second-stage juveniles (infective stage) penetrate root tips and migrate to the vascular cylinder, in which RKNs interact with the host plant. In susceptible host plants, RKNs induce a series of complicated changes in the plant cell architecture. 
Secondary metabolites in plants are also important for repelling and attracting RKNs. Although RKNs have an extensive host range, host plant species have significantly different levels of attractiveness for different RKN species (Wang et al. 2009). Although both the attractants and repellents for RKNs remain largely unknown, the basal levels or types of attractants and repellents differ according to the host species. Previous studies have suggested that JA and ET may participate in the attractiveness of plants to RKNs because the efficiency or degree of RKN penetration was shown to be changed in Arabidopsis or tomato mutants deficient in JA or ET signaling (Bhattarai et al. 2008; Fudali et al. 2013; Fujimoto et al. 2011a,b). JA and ET are involved in modulating herbivore-induced metabolite emissions (Schmelz et al. 2003). For example, the activation of ET biosynthesis led to the production of defense compounds such as antimicrobial substances (Pedras et al. 2000). Sclareol enhanced the expression of the Arabidopsis $\mathrm{ABC}$ transporter gene AtPDR12, the induction of which required activation of the ET signaling pathway (Campbell et al. 2003).

In the course of exploring the plant secondary metabolites involved in attracting or repelling RKNs, we found that sclareol inhibited its penetration. Here, we demonstrated that sclareol conferred resistance to RKN penetration by partially activating ET-dependent defense responses.

\section{RESULTS}

\section{Exogenous application of sclareol inhibits penetration of RKNs Into tomato roots.}

To examine the effect of sclareol (Fig. 1) on the penetration of RKNs into plant roots, we used tomato, an extremely susceptible host plant for RKNs. The roots of 3-week-old tomato plants grown in sand in pots treated with different concentrations $(50,80,100$, or $200 \mu \mathrm{M})$ of sclareol, incubated for $48 \mathrm{~h}$, inoculated with RKNs, and stained with acid fuchsin, an agent that stains nematodes, 7 days after inoculation. The degree of inhibition of RKN penetration was estimated by counting the number of stained RKNs. The penetration of RKNs was inhibited by sclareol at 100 or $200 \mu \mathrm{M}$ to approximately $30 \%$ of the control methanol treatment (Fig. 2A). To determine the duration of treatment required to manifest the inhibitory effect of sclareol, tomato roots treated with $100 \mu \mathrm{M}$ sclareol were incubated for 24,48 , or $72 \mathrm{~h}$ and assayed for RKN resistance. Treatment for $24 \mathrm{~h}$ had no effect on inhibition of RKN penetration, whereas treatment for $48 \mathrm{~h}$ or longer had an inhibitory effect (Fig. 2B). Based on these results, we used sclareol at a concentration of $100 \mu \mathrm{M}$ and with a treatment period of $48 \mathrm{~h}$ for further analyses. To gain insight into its structure-activity relationship, we assayed sclareolide (Fig. 1), a naturally occurring sesquiterpene lactone with a chemical structure similar to sclareol. Sclareolide did not have any inhibitory activity for penetration of RKNs into tomato roots (Fig. 2C and D).

To determine whether inhibition of RKN penetration by sclareol was attributed to its nematicidal activity, the effect of sclareol on the survival of RKNs was tested. Sclareol had little or no effect on the survival of RKNs (Supplementary Table S1). Whether sclareol affected the infectivity of RKNs was also examined by inoculating a suspension of RKNs that had been previously incubated with sclareol, or methanol as a control, for $48 \mathrm{~h}$ on tomato roots, followed by counting the number of RKNs penetrated into the tomato roots (Supplementary Fig. S1A). No significant difference was observed in the number of RKNs between treatments (Supplementary Fig. S1B).

\section{EIN2-dependent ET signaling is involved in inhibition of RKN penetration by sclareol.}

The results from the RKN penetration assays using tomato (Fig. 2) suggested that inhibition of RKN penetration by sclareol is due to defense responses induced or enhanced in the host plant by the chemical. As the first step to identify host factors involved in signaling of such defense responses, we analyzed the expression of known marker genes related to phytohormone signaling in Arabidopsis, a model plant for studying plantnematode interactions, after treatment with sclareol. Arabidopsis plants were grown in two different growth conditions, and plants grown in a sand system were used for gene expression analysis (Fig. 3A). In addition, plants were grown in a hydroponic system, which provided intact roots for other biochemical and histochemical analyses. Roots of wild-type (WT) Columbia (Col-0) plants grown in the sand system were treated with sclareol, and the expression levels of marker genes in the treated roots were examined by real-time reverse-transcriptase polymerase chain reaction (RT-PCR). Sclareol enhanced the accumulation of transcripts for the JA/ET signaling marker gene PDF1.2., whereas it had little or no effect on the accumulation of transcripts for $c h i B$, another JA/ET signaling marker gene (Fig. 3B). None of the SA signaling marker genes (BGL2 and $P R 1)$ or the JA signaling marker genes (VSP2 and $L O X 2$ ) responded to sclareol. Quantitative analysis of endogenous SA and JA in roots also showed that neither SA nor JA levels were changed by treatment with sclareol (Supplementary Fig. S2).

To further examine which phytohormone is involved, Arabidopsis mutants defective in the perception of specific phytohormones were assayed for resistance to RKN penetration. We first confirmed whether exogenously applied sclareol inhibits the penetration of RKNs into Arabidopsis roots using WT plants grown in the sand system. The number of RKNs penetrated into sclareol-treated roots was approximately half of that into methanol-treated roots (Fig. 4, WT). We next assayed using the SA signaling mutant NONEXPRESSER OF PATHOGENESIS-RELATED GENES 1-1 (nprl-1), an abscisic acid (ABA) signaling mutant ABA-INSENSITIVE1-1 (abil-1), a JA signaling mutant CORONATINE-INSENSITIVE1-1 (coil1), and an ET signaling mutant ETHYLENE-INSENSITIVE2-1 (ein2-1). In nprl-1 and abil-1, sclareol reduced the number of RKNs that penetrated into the roots to the same degree as shown in WT plants (Fig. 4). coil-1 exhibited relatively low susceptibility to infection, as indicated by decreased penetration of RKNs in the absence of sclareol compared with WT plants, and there was no difference in the number of RKNs that penetrated into roots between treatments in this mutant. Inhibition of RKN penetration by sclareol was not observed in ein2-1. These results suggested that EIN2 is involved in sclareol-induced resistance to RKN penetration.

\section{Lignin is involved in EIN2-mediated RKN resistance induced by sclareol.}

To identify the factors that function downstream of EIN2 in sclareol-induced RKN resistance, we used microarray data on sclareol-responsive Arabidopsis root genes (Seo et al. 2012). Our previous microarray analysis identified many genes with a

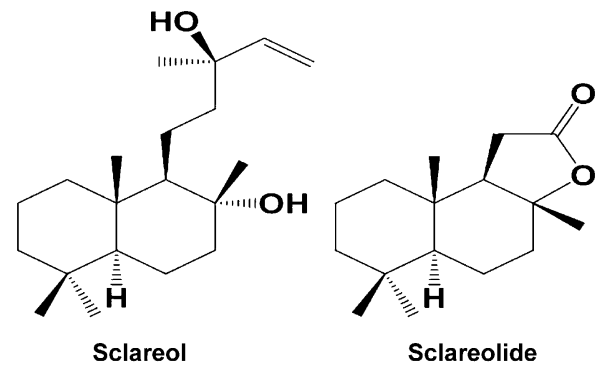

Fig. 1. Chemical structures of sclareol and sclareolide. 
role in phytohormone responses, defense responses, and metabolism. Among them, we examined the possibility of genes for lignin biosynthetic enzymes and ET-responsive factors (ERFs) as such downstream factors, because lignin plays a role in defenses in roots and because ERFs function in ET signaling. If these genes function downstream of EIN2, it is expected that their expression would not be induced by sclareol in the ein2-1 mutant. Roots of ein2-1 mutant plants grown in the sand system were treated with sclareol or methanol, and the induction kinetics were assessed for three lignin biosynthetic genes-phenylalanine ammonia-lyase $(P A L 1)$, trans-cinnamate 4-monooxygenase $(\mathrm{C} 4 \mathrm{H})$, and cinnamoyl-CoA reductase $(C C R 2)-$ and an ERF gene $(E R F 2)$. There was no difference in the degree of accumulation of transcripts for $P A L 1, C 4 H$, and $C C R 2$ between treatments in ein2-1, whereas accumulation of transcripts for these three genes was enhanced by sclareol in WT plants (Fig. 5, ein2-1 and WT). To confirm whether the behavior of PAL1, C4H, and $C C R 2$ observed in ein2-1 was specific to this mutant, their induction kinetics in other phytohormone-related mutants were also examined. In nprl-1, abil-1, and coil-1, as in WT plants, the PAL1, C4H, and CCR2 genes responded substantially to sclareol. Accumulation of transcripts for ERF2 was enhanced by sclareol in the WT but not in ein2-1 (data not shown). We also checked the expression of AtPDR12, a gene that was previously identified as the most highly induced gene by microarray analysis (Seo et al. 2012), in ein2-1 after treatment with sclareol. The degree of AtPDR12 transcript accumulation was almost equal between treatments in ein2-1. This was consistent with a previous report showing that EIN2 regulates the expression of AtPDR12 (Campbell et al. 2003).

The result shown in Figure 5 suggested the possible involvement of lignin in EIN2-mediated RKN resistance induced by sclareol. To further test the possibility, we first examined whether sclareol induced accumulation of lignin. Roots of WT plants grown in the sand system were treated with sclareol, and the endogenous content of thioglycolic acid lignin in the treated roots was determined. Sclareol increased thioglycolic acid lignin levels by approximately threefold compared with the control methanol treatment (Fig. 6A, WT). Such an increase was also detected in roots of the nprl-1, abil-1, and coil-1 mutants. In contrast, roots of ein2- 1 exhibited no increase in thioglycolic acid lignin in response to sclareol. To analyze the localization of lignin in roots, roots of hydroponically grown plants were treated with sclareol and stained with phloroglucinol, an agent that stains lignin. In sclareol-treated roots of WT plants, lignin staining was detected in the root hair zone in the root tip (Fig. 6B, sclareol in WT). Such staining was undetectable in the corresponding zone in ein2-1 (Fig. 6B, sclareol in ein2-1). We also examined the effect of 1-aminocyclopropane-1-carboxylic acid (ACC), the biosynthetic precursor of ET, on accumulation of lignin. Exogenously applied ACC induced the accumulation of lignin in roots of WT but not ein2-1, although the degree of induction was lower than that by sclareol (Fig. 6B, ACC in WT and ein2-1).

Finally, we assessed whether lignin accumulation was necessary for sclareol-induced inhibition of RKN penetration using an Arabidopsis mutant for CCR2, ccr2 (Nossen background). Quantitative analysis of lignin confirmed that roots of $c c r 2$ failed to accumulate thioglycolic acid lignin in response to sclareol, whereas roots of the WT with the same genetic background did (Fig. 6C). Roots of $c c r 2$ exhibited, in response to sclareol, no inhibition of RKN penetration (Fig. 6D) and no enhancement of $P A L 1, C 4 H$, and $C C R 2$ transcript accumulation (Supplementary Fig. S3).

Activation of ET biosynthesis is also important for sclareol-induced RKN resistance.

The involvement of ET biosynthesis in sclareol-induced RKN resistance was also investigated. We first examined
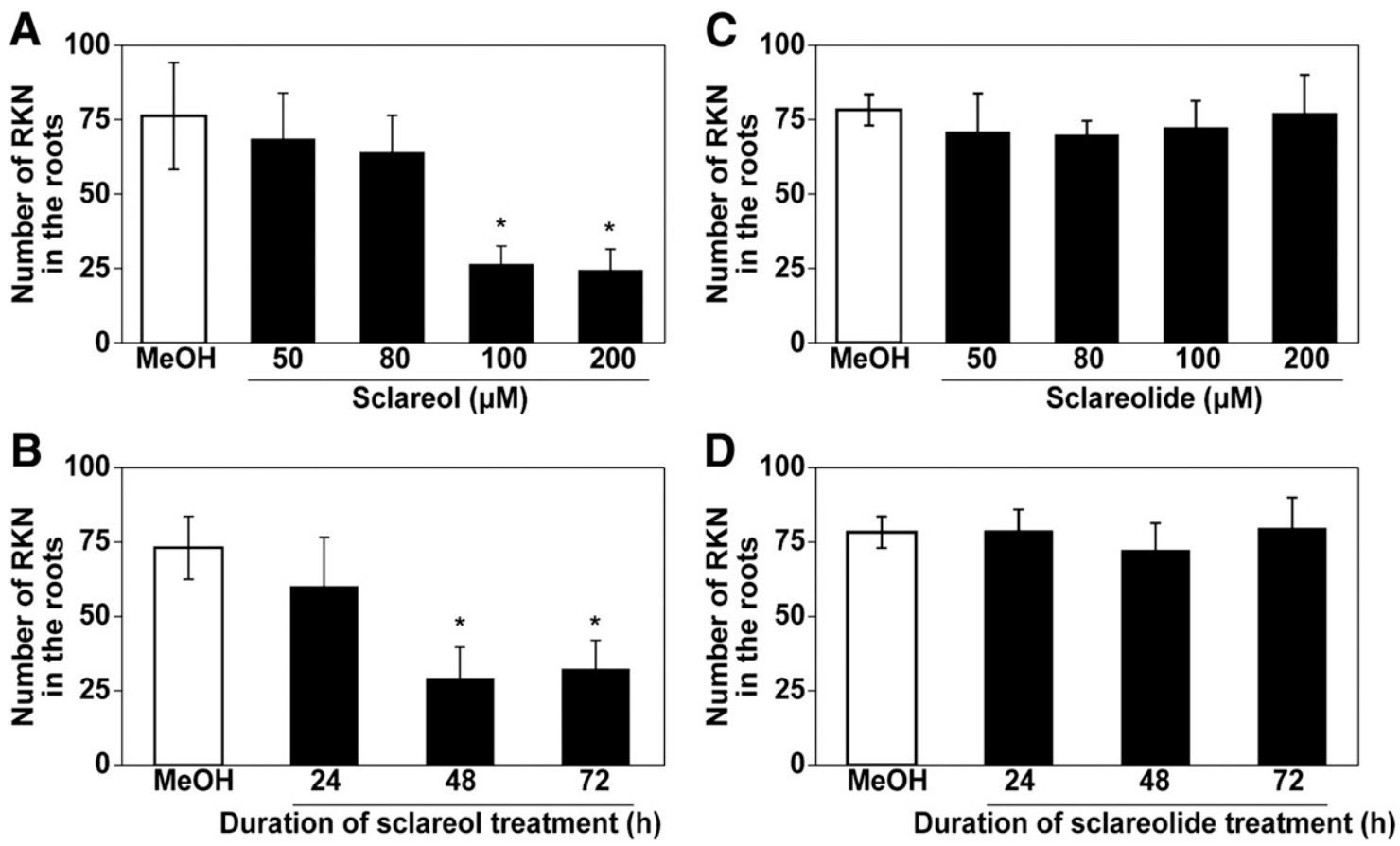

Fig. 2. Effect of sclareol and sclareolide on the penetration of root-knot nematodes (RKNs) into tomato roots. A and C, Dose-dependent effect of sclareol and sclareolide, respectively. Tomato roots pretreated with the indicated concentrations of sclareol for $48 \mathrm{~h}$ were inoculated with RKNs, and the number of RKNs that penetrated into the roots was counted 7 days after inoculation. B and D, Tomato roots pretreated with $100 \mu \mathrm{M}$ sclareol or sclareolide for 24,48 , or $72 \mathrm{~h}$ were inoculated with RKNs, and the number of RKNs that penetrated into the roots was counted 7 days after inoculation. A to D, Methanol (MeOH) $(0.1 \%)$ was used as a control. Values are the mean \pm standard deviation from two independent experiments, each performed in five replicates. Asterisks denote statistically significant differences compared with the $0.1 \% \mathrm{MeOH}$ control (Tukey's honestly significant difference test; $P<0.05$ ). 
whether sclareol activates ET biosynthesis by measuring endogenous amounts of ET in WT Arabidopsis plants after treatment with sclareol. Because of the difficulty in measuring ET from roots without applying any wound stresses that allow its biosynthesis to activate, hydroponically grown WT plants were sealed in a test tube, with the roots submerged in a solution containing sclareol, and ET released from the whole plant was analyzed. Plants treated with sclareol produced 3.5-fold more ET than plants treated with methanol (Fig. 7A). Sclareolide caused only a slight increase in ET emission (Supplementary Fig. S4). Consistent with enhanced ET biosynthesis, accumulation of transcripts for the ACC synthase (ACS) genes ACS2 and ACS6 in WT roots was enhanced by sclareol (Fig. 7B). These results suggested that sclareol activates biosynthesis of ET.

Stress-induced activation of ET biosynthesis requires activation of mitogen-activated protein kinases (MAPKs) in plants. In Arabidopsis, external stimuli such as wounding cause a rapid activation of two MAPKs, MPK3 and MPK6, which results in triggering biosynthesis of ET through phosphorylation of stress-responsive ACS such as ACS2 and ACS6 by the activated MPK3 and MPK6 (Li et al. 2012). We examined whether MPK3 and MPK6 are involved in sclareol-induced RKN resistance. MAPK activity can be detected by measuring the phosphotransferase activity for the target substrate in an immune complex between the MAPK and a MAPK-specific antibody. To detect the activities of MPK3 and MPK6, we generated anti-MPK3 and anti-MPK6 antibodies against antigen peptides corresponding to the $\mathrm{C}$-terminal amino acid regions of each MAPK (see Materials and Methods for details). The specificity of these antibodies was assessed by determining the enzymatic activities of MPK3 and MPK6 in mpk3-2 and mpk6-4 (Arabidopsis mutants for MPK3 and MPK6, respectively) after wounding. Total extracts from

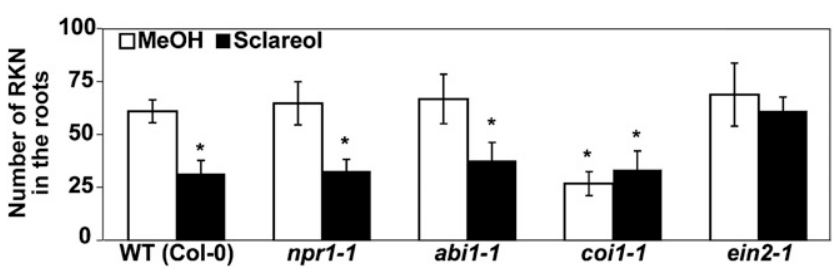

Fig. 4. Effect of sclareol on penetration of root-knot nematodes (RKNs) into roots of defense-related Arabidopsis mutants. Roots of wild-type (WT) (Col-0) or mutant plants pretreated with $100 \mu \mathrm{M}$ sclareol for $48 \mathrm{~h}$ were inoculated with RKNs, and the number of RKNs that penetrated into the roots was counted 7 days after inoculation. Values are the mean \pm standard deviation from two independent experiments, each performed in five replicates. Asterisks denote statistically significant differences compared with the $0.1 \%$ methanol $(\mathrm{MeOH})$ control of the WT (Tukey's honestly significant difference test; $P<0.05)$.
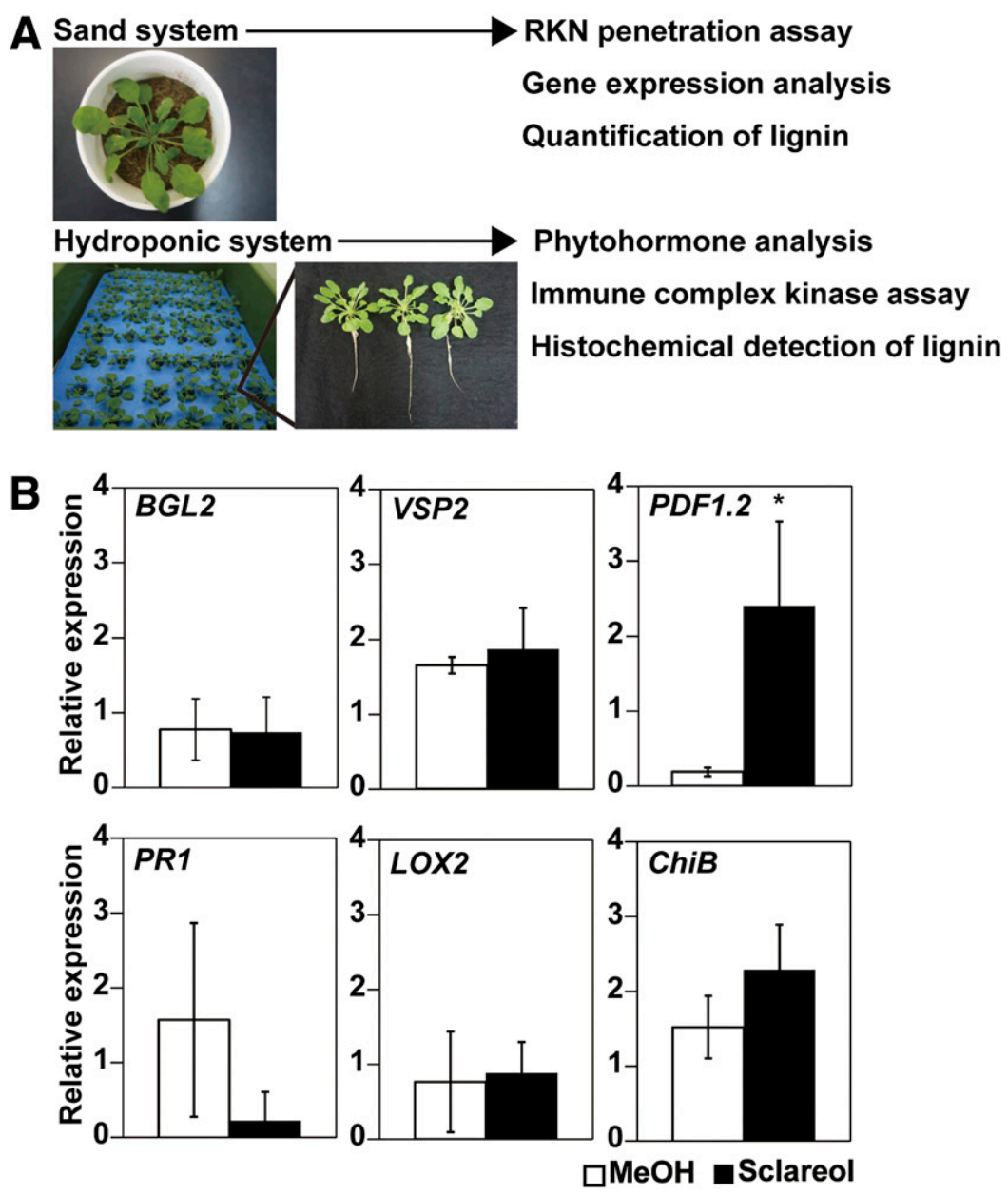

Fig. 3. Analysis of expression of defense-related genes in Arabidopsis roots after treatment with sclareol. A, Plant growth systems used. B, Real-time reversetranscriptase polymerase chain reaction analysis of salicylic acid (SA)- (BGL2 and PR-1), jasmonic acid (JA)- (VSP and LOX2), and JA/ethylene (ET)responsive $(P D F 1.2$ and $C h i B)$ genes in wild-type (Col-0) roots $48 \mathrm{~h}$ after treatment with $100 \mu \mathrm{M}$ sclareol or $0.1 \%$ methanol $(\mathrm{MeOH})$. Values are the mean \pm standard deviation from three independent measurements. Asterisks denote statistically significant differences compared with the $0.1 \%$ MeOH control (Student $t$ test $P<0.05)$ 
wounded leaves of $m p k 3-2$ or $m p k 6-4$ mutant plants grown in the hydroponic system were immunoprecipitated with antiMPK3 or anti-MPK6 antibodies, and kinase activities in the immune complexes using myelin basic protein (MBP) as an artificial substrate were determined. Little wounding-induced activity of MPK3 was detected in $m p k 3-2$, whereas little wounding-induced activity of MPK6 was detected in $m p k 6-4$ (Supplementary Fig. S5). Immunoblotting analysis indicated
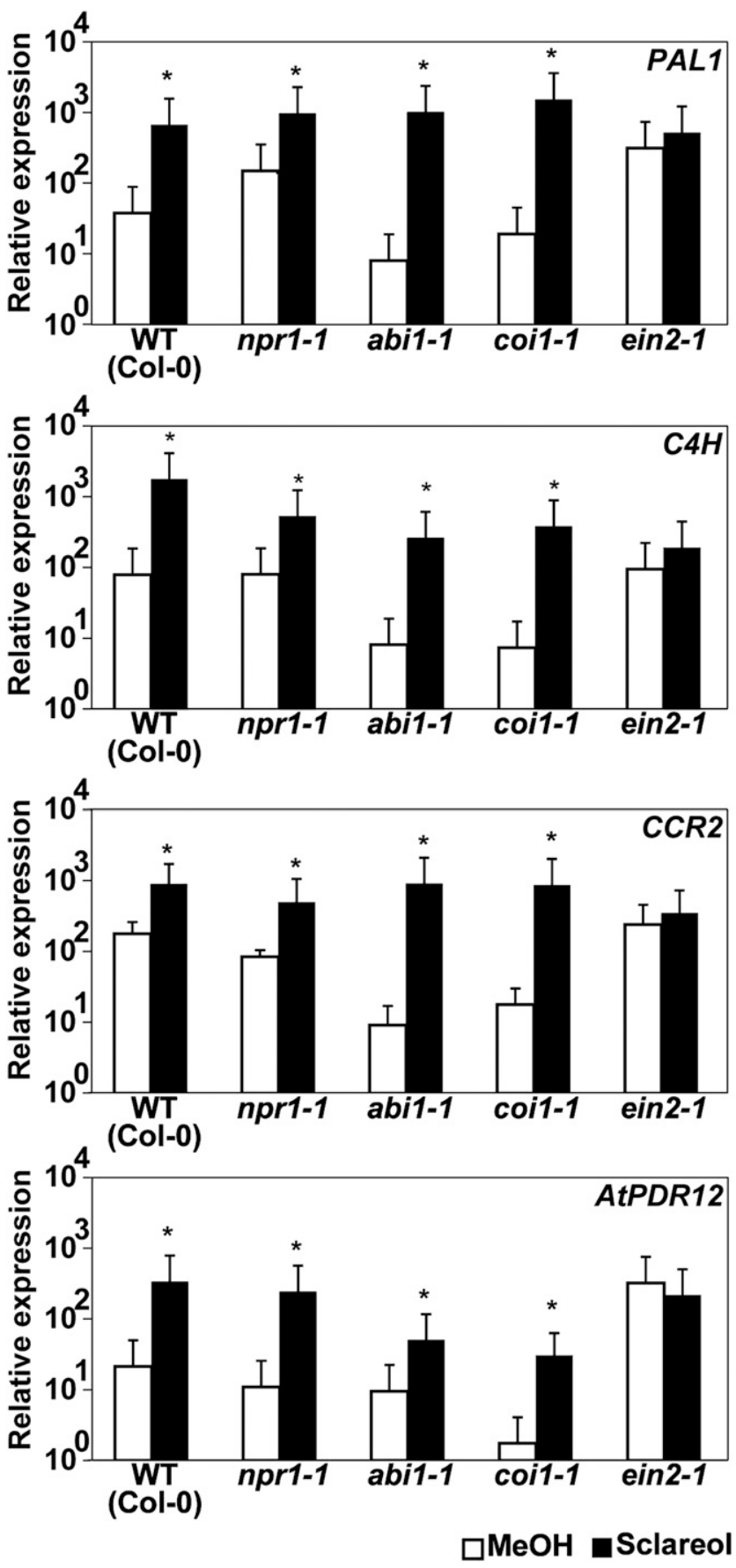

Fig. 5. Gene expression analysis of roots of defense-related Arabidopsis mutants after treatment with sclareol. Real-time reverse-transcriptase polymerase chain reaction analysis of defense-related genes in roots of wild-type (WT) (Col-0) and mutants $48 \mathrm{~h}$ after treatment with $100 \mu \mathrm{M}$ sclareol or $0.1 \%$ methanol $(\mathrm{MeOH})$. Values are the mean \pm standard deviation from three independent measurements. Asterisks denote statistically significant differences compared with the MeOH control (Student $t$ test; $P<$ $0.05)$. Each value represents the relative degree of expression. that a protein that reacted with the anti-MPK3 antibody was undetectable in $m p k 3-2$, whereas a protein that reacted with the anti-MPK6 antibody was undetectable in mpk6-4. Thus, the anti-MPK3 and anti-MPK6 antibodies we generated can be used to specifically detect the activities of MPK3 and MPK6, respectively.

To examine whether exogenously applied sclareol activates MPK3 and MPK6 in roots, we used plants grown in the hydroponic system because pulling the root out of the pot gives the plant a wound that causes rapid activation of MAPKs (Rodriguez et al. 2010). Roots of hydroponically grown WT plants were treated with sclareol and subjected to immunoprecipitation with anti-MPK3 or anti-MPK6 antibodies followed by the immune complex MBP kinase assay. The MBP kinase activities of MPK3 and MPK6 began to increase 5 min after treatment, reached a peak at $30 \mathrm{~min}$, and thereafter returned to the basal level (Fig. 7C). Methanol treatment did not increase the kinase activities of these two MAPKs. The protein levels of MPK3 and MPK6 were almost equal over the time course, suggesting that rapid activation of MPK3 and MPK6 by sclareol occurs at the posttranslational level.

Neither mpk3-2 nor mpk6-4 exhibited inhibition of RKN penetration in response to sclareol (Fig. 7D). We also examined whether ACC possesses the same inhibitory effect on RKN penetration as sclareol. When roots of WT Arabidopsis grown in the sand system were treated with $50 \mu \mathrm{M}$ ACC and inoculated with RKNs, the number of penetrating RKNs was decreased compared with methanol-treated roots, although the inhibitory effect was smaller than sclareol treatment (Fig. 7D, sclareol versus ACC in WT). None of genes ein2-1, mpk3-2, or mpk6-4 exhibited inhibition of RKN penetration in response to ACC.

\section{DISCUSSION}

Exogenously applied sclareol did not show any nematicidal activity for RKNs or alter their infectivity, suggesting that inhibition of RKN penetration by sclareol was due to host defense responses elicited or enhanced in the plant after treatment with this chemical. Several lines of evidence demonstrate that a signal transduction pathway leading to such host responses is mediated by ET and its downstream factor, EIN2. First, ein2-1 exhibited a complete absence of inhibition of RKN penetration by sclareol (Fig. 4). Second, sclareol enhanced both the production of endogenous ET and the expression of ET-responsive or biosynthetic genes (Fig. 7A and B). Third, sclareol activated MPK3 and MPK6 (Fig. 7C), MAPKs whose activation is required for triggering ET biosynthesis. Fourth, neither $m p k 3$ nor mpk6 exhibited inhibition of RKN penetration in response to sclareol (Fig. 7D). These results suggested that, in roots of Arabidopsis and, possibly, tomato, exogenously applied sclareol activates MPK3 and MPK6 which, in turn, triggers biosynthesis of ET.

Invasion of pathogens, such as viruses, bacteria, and fungi, enhances ET biosynthesis in plants (Wang et al. 2002). Plantparasitic nematodes also elicited elevated levels of endogenous ET in plant roots (Glazer et al. 1983, 1985; Volkmar 1991). Fudali and associates (2013) reported that, compared with WT Arabidopsis, ET-overproducing mutants (eto1, eto2, and eto3) were less attractive to RKNs (Meloidogyne hapla), whereas an ET-insensitive mutant (ein2-1) was more attractive to the same nematode. In plant-RKN interactions, ET may play a positive role in resistance to RKNs. However, the ET biosynthesis and signaling pathways seemed not to be the sole pathway that led to inhibition of RKN penetration, because the inhibitory effect of exogenously applied ACC, a direct biosynthetic precursor of ET, on RKN penetration was lower than that of sclareol 
(Fig. 7D). It is likely that activation of ET biosynthesis and signaling pathways is necessary but not sufficient to inhibit of RKN penetration. Sclareol does not necessarily activate the whole ET signaling pathway, because $c h i B$, an ET-responsive gene, did not respond to sclareol (Fig. 3B). It remains unclear whether the ET signaling pathway that is not activated by sclareol is involved in resistance to $\mathrm{RKN}$, and further studies to resolve this issue are required.

SA is unlikely to be involved in sclareol-induced resistance because npr1-1, an SA-signaling mutant, exhibited enhanced sclareol-induced inhibition of RKN penetration and because treatment with sclareol did not alter endogenous SA content or expression of SA-responsive genes. Such inhibition was also abolished in the coil-1 mutant, a JA-signaling mutant. However, this abolition might be due to the phenotypic profile of reduced susceptibility of this mutant to RKN penetration. It has been shown that RKNs need an intact JA pathway when they parasitize a host plant (Bhattarai et al. 2008; Fujimoto et al. 2011b). The results that sclareol did not alter endogenous JA content or expression of JA-responsive genes suggested that JA is not involved in sclareol-induced resistance.
There are two possibilities to explain a reduction in RKN penetration in roots treated with sclareol. One possibility is that lignin induced by sclareol is partially involved in inhibiting penetration of RKNs into roots. Lignin is synthesized via the phenylpropanoid pathway and its synthesis has been shown to be regulated at the transcriptional level (Raes et al. 2003). Sclareol enhanced both expression of the phenylpropanoid pathway genes PAL1, C4H, and CCR2 and accumulation of lignin. In general, RKNs penetrate into meristematic and elongation zones in the root tip (Wyss et al. 1992). We could not detect lignin staining at such sites of RKN penetration; lignin staining was detected mainly in the root hair zone behind the elongation zone (Fig. 6B). It is possible that accumulation of lignin occurred in the meristematic and elongation zones in response to sclareol but the accumulation failed to be detected histochemically due to a relatively low detection limit of the staining method we used. The histochemical localization of lignin did not correlate with the site of RKN penetration in our experimental conditions. Nevertheless, our results from the RKN penetration assay using $c c r 2$, a mutant defective in lignin accumulation, indicated the importance of lignin in sclareol-induced RKN
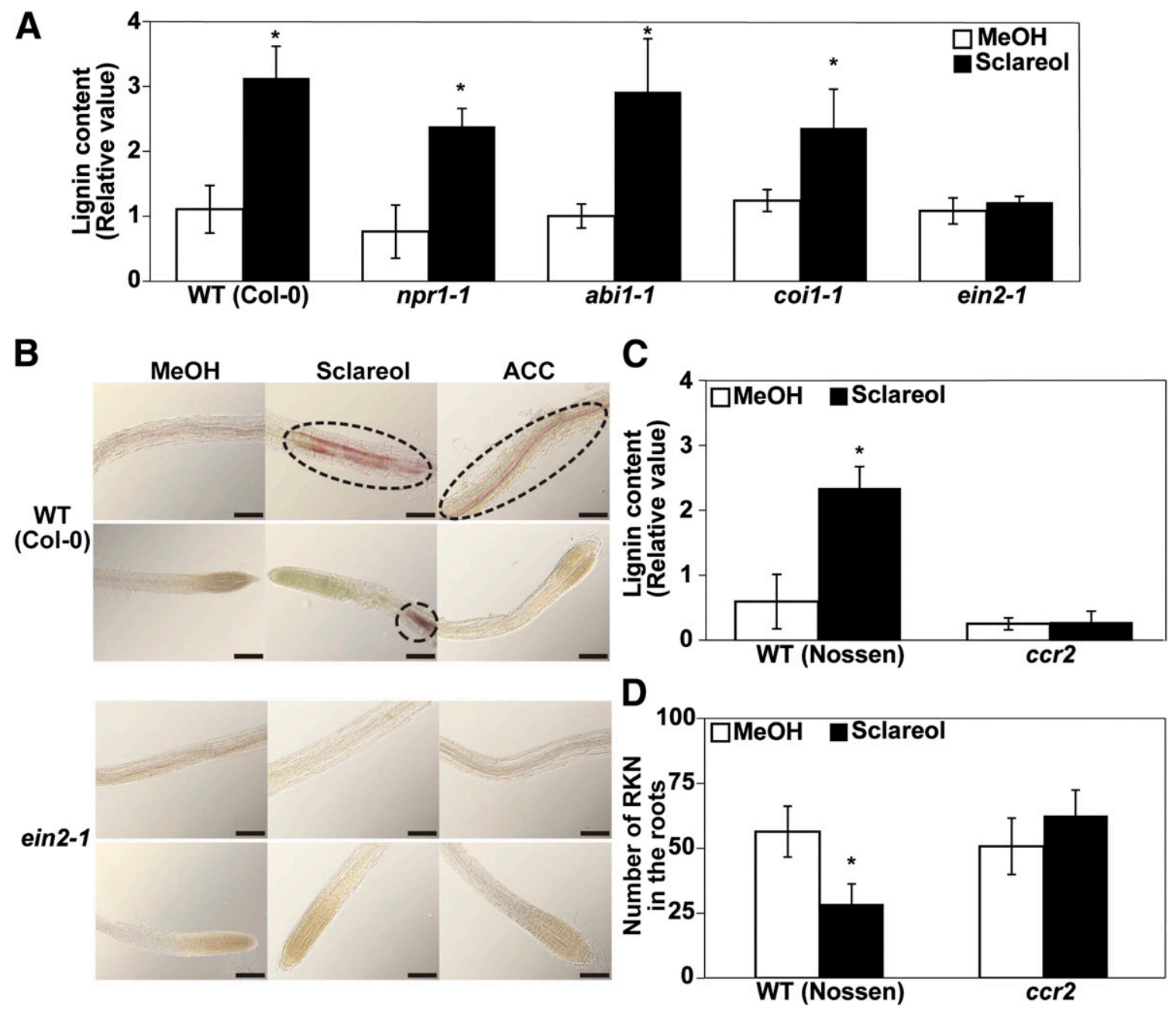

Fig. 6. Analysis of lignin accumulation and root-knot nematode (RKN) penetration in Arabidopsis roots treated with sclareol. A, Thioglycolic acid lignin concentrations in roots of wild-type (WT) (Col-0) and defense-related mutants 3 days after treatment with $100 \mu \mathrm{M} \mathrm{sclareol} \mathrm{or} 0.1 \% \mathrm{methanol}(\mathrm{MeOH})$. B, Histochemical localization of lignin in the root tips of WT (Col-0) and an ein2-1 mutant 3 days after treatment with $100 \mu \mathrm{M}$ sclareol or $50 \mu \mathrm{M}$ 1-aminocyclopropane-1-carboxylic acid (ACC). Dashed lines indicate where lignin was detected. Bars indicate $100 \mu \mathrm{m}$. C, Thioglycolic acid lignin concentrations in roots of WT (Nossen) and $c c r 2$ mutant 3 days after $100 \mu \mathrm{M}$ sclareol. D, Roots of WT (Nossen) or $c c r 2$ mutant plants pretreated with $100 \mu \mathrm{M}$ sclareol for $48 \mathrm{~h}$ were inoculated with RKNs, and the number of RKNs that penetrated into the roots was counted 7 days after inoculation. Values are the mean \pm standard deviation (SD) from two independent experiments, each performed in five replicates. $\mathrm{MeOH}(0.1 \%)$ was used as a control. A and C, Values are the mean \pm SD from five replicates. A, C, and D, Asterisks denote statistically significant differences compared with the $0.1 \%$ MeOH control of the WT (Tukey's honestly significant difference test; $P<0.05$ ). 
resistance. Lignin produced in response to sclareol may function as a physical barrier for the invasion of RKNs. Because sclareol-induced enhancement of lignin accumulation was abolished in ein2-1, it is likely that lignification induced by sclareol occurs downstream of EIN2.

Another possibility is a partial involvement of metabolites induced by sclareol. Sclareol enhanced the expression of many genes, including $P A L 1$, related to biosynthesis of secondary metabolites such as flavonoids, alkaloids, and phenolics (Fig. 5) (Campbell et al. 2003; Grec et al. 2003; Jasiński et al. 2001; Seo et al. 2012). Flavonoids are known to act as repellents for nematodes and inhibit their motility and hatching (Wuyts et al. 2006). It is possible that roots produce metabolites in response to sclareol, and some of them function as inhibitors for RKN penetration.

The phenomena observed in the present study were similar to those in a previous study with the combination of Arabidopsis and $R$. solanacearum (Seo et al. 2012); exogenously applied sclareol conferred resistance to $R$. solanacearum without exhibiting any antibacterial activity, and the resistance required EIN2 and MPK3. These findings suggested that defense mechanisms induced by sclareol are extremely common in many plant species. However, there are few plant species that
A

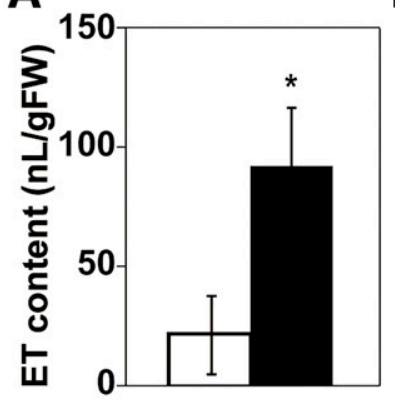

B

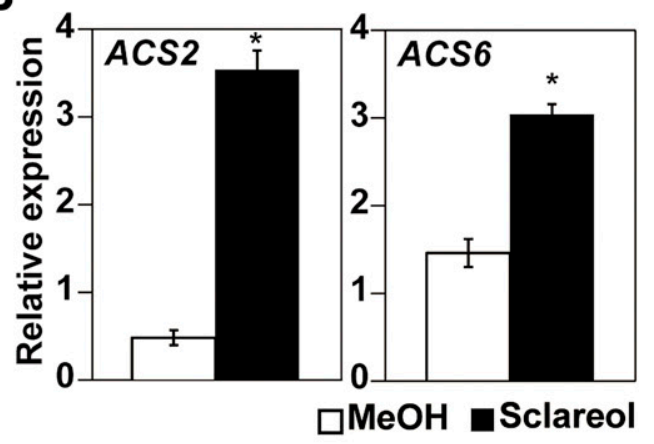

C

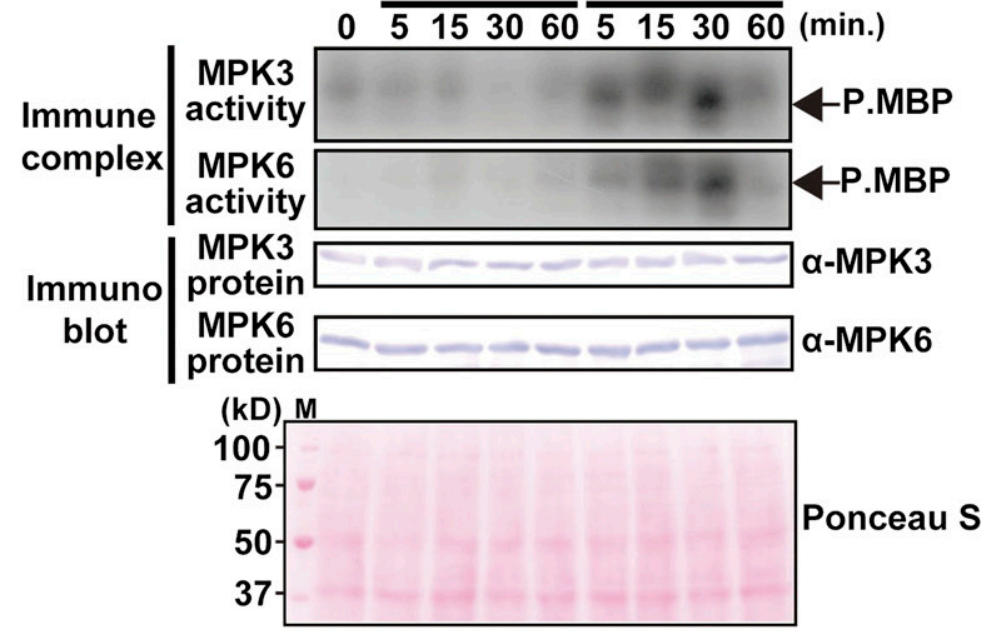

D

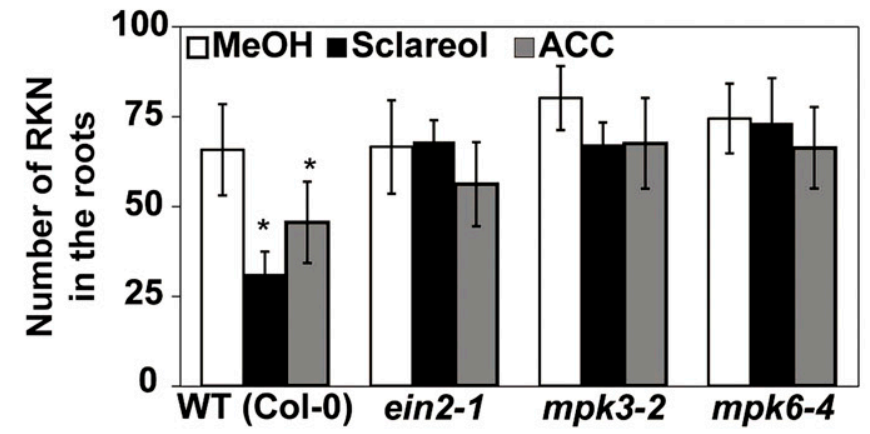

Fig. 7. Analysis of importance of ethylene (ET) biosynthesis in sclareol-induced root-knot nematode (RKN) resistance. A, Concentrations of ET emitted from wild-type (WT) (Col-0) plants $48 \mathrm{~h}$ after treatment with $100 \mu \mathrm{M}$ sclareol. B, Real-time reverse-transcriptase polymerase chain reaction analysis of ET biosynthetic genes in WT (Col-0) roots $48 \mathrm{~h}$ after treatment with $100 \mu \mathrm{M}$ sclareol or $0.1 \%$ methanol (MeOH). A and $\mathbf{B}$, Values are the mean \pm standard deviation (SD) from three independent measurements. C, Analysis for immune complex myelin basic protein (MBP) kinase activity of MPK3 and MPK6 and immunoblotting analysis of their protein levels using anti-MPK3 ( $\alpha$-MPK3) or anti-MPK6 ( $\alpha$-MPK6) antibodies. Arrows indicate phosphorylated myelin basic protein (P.MBP). Ponceau S staining is shown for equal loading of samples. M indicates a molecular marker. D, Roots of WT (Col-0) or mutant plants pretreated with $100 \mu \mathrm{M}$ sclareol, $50 \mu \mathrm{M}$ $\mathrm{ACC}$, or $0.1 \% \mathrm{MeOH}$ for $48 \mathrm{~h}$ were inoculated with RKNs, and the number of RKNs that penetrated into the roots was counted 7 days after inoculation. Values are the mean \pm SD from two independent experiments, each performed in five replicates. A, B, and D, Asterisks denote statistically significant differences compared with the $0.1 \% \mathrm{MeOH}$ control of WT (Student's $t$ test for A and B, $P<0.05$; Tukey's honestly significant difference test for D, $P<0.05$ ). 
produce sclareol, and both tomato and Arabidopsis, the plant species used in this study, do not produce sclareol. Therefore, sclareol is not an endogenous signaling substance that mediates defense responses in tomato and Arabidopsis but an elicitorlike substance that activates such responses. Further studies, including the identification of metabolites induced by sclareol, will help us to understand the mechanisms of plant defense against nematodes and pathogens.

\section{MATERIALS AND METHODS}

\section{Plant material and growth conditions.}

Seed of tomato (Solanum lycopersicum 'Momotaro'), which carries the Mi-gene (Milligan et al. 1998; Nombela et al. 2003), was purchased from a seed company (Takii \& Co. Ltd., Kyoto, Japan). Seedlings (7 to 10 days old) were transferred to autoclaved sand with an average particle size of $0.3 \mathrm{~mm}$ in diameter, packed in $220-\mathrm{ml}$ plastic pots, and grown at 23 to $27^{\circ} \mathrm{C}$ with $16 \mathrm{~h}$ of light. A commercial liquid fertilizer (Hyponex) diluted 3,000-fold with water was applied to the pots every 3 days. Unless otherwise stated, tomato plants with two true leaves that had fully expanded were used.

Arabidopsis thaliana with Columbia (Col-0) and Nossen backgrounds was used. All the mutants except for $c c r 2$ were in the Col-0 background. coil-1, nprl-1, abil-1, and ein $2-1$ have been described previously (Abe et al. 2008; Leon-Reyes et al. 2009; Uno et al. 2000), as have mpk3-2 and mpk6-4 (Seo et al. 2012). ccr2 (resource number pst20225), a transposon-tagged mutant line of CCR2 gene in a Nossen background, was provided by the RIKEN BioResource Center.

Because coil-1 is male sterile (Xie et al. 1998), an $F_{2}$ population segregating for this mutation was used to select homozygous coil-1 plants. The coil-1 mutant has a single base substitution at nucleotide position 1,663 , resulting in conversion to a nucleotide sequence of GAATGCN, which is recognized by the restriction enzyme BsmI (Xie et al. 1998). For selection, genomic DNA purified from a single rosette leaf of each plant was amplified using DNA polymerase (ExTaq, Takara Bio, Shiga, Japan) and the set of primer 5'-CTATCT GAGACAAGGCGGCTTAACCGAC-3' and 5'-CAATTTTGT AACCGCTGCAGCGATTGCTCG- ${ }^{\prime}$. After digesting with $B s m I$, the reaction mixture was analyzed by agarose gel electrophoresis. The plants in which digested bands were observed were regarded as homozygous coil-1 plants.

All Arabidopsis plants were grown in either the sand system or the hydroponic system. For the sand system, 3-week-old seedlings were transferred individually to autoclaved sand with an average particle size of $0.3 \mathrm{~mm}$ in diameter, packed in $220-\mathrm{ml}$ plastic pots, and grown at $22^{\circ} \mathrm{C}$ with $16 \mathrm{~h}$ of light. Hyponex, diluted 3,000-fold, was applied to the pots every 5 to 7 days. Ten-week-old plants were used for each analysis.
For the hydroponic system, seed were sown on half-strength Murashige and Skoog medium (Wako Pure Chemical, Osaka, Japan) supplemented with $0.7 \%$ agar and grown at 20 to $22^{\circ} \mathrm{C}$ with $8 \mathrm{~h}$ of light. Seedlings (10 to 14 days old) were grown on a polyethylene raft through holes $(1 \mathrm{~cm}$ in diameter) floating on 3,000-fold diluted Hyponex that was constantly aerated with an air pump, with their roots submerged in the fertilizer. For each analysis, 8- to 10 -week-old plants were used.

\section{Preparation and maintenance of nematodes.}

A strain (collection number 108258) of RKNs (M. incognita), which possesses the RKN resistance gene $M i$, was provided from the MAFF gene bank of the National Institute of Agrobiological Sciences of Japan and has been used in previous studies (Fujimoto et al. 2011a,b). Maintenance and preparation of the strain were in accordance with the procedure described by Fujimoto and associates (2011a). RKNs collected 7 to 10 days after hatching were used for each analysis.

\section{Chemical treatments.}

Stock solutions (100 $\mathrm{mM})$ of sclareol (Sigma) and sclareolide (Sigma) in methanol and a stock solution $(100 \mathrm{mM})$ of ACC (Sigma) in water were diluted in water to various concentrations. Methanol concentrations did not exceed $0.1 \%$ in all the experiments.

Roots of plants grown in the sand system were treated by soaking the pot itself in a solution containing various concentrations of each chemical in a tray, with their roots submerged in the solution. After soaking for 24,48 , or $72 \mathrm{~h}$, the treated plants were transferred to a new tray containing water and used for RKN penetration assays; or the roots were pulled out of the pots, washed with water, and used for real-time RT-PCR analysis or quantification of thioglycolic acid lignin.

For treatment of the roots of hydroponically grown plants, plants were pulled out of the fertilizer, transferred to a petri dish containing water with their roots submerged in the water, and preincubated for $48 \mathrm{~h}$ to diminish the influence of any wound stresses that could be caused by removal. After discarding water using a pipette, the plant roots were treated by gently adding a solution containing various concentrations of each chemical to the petri dish until the roots were submerged in the solution, incubated for adequate time periods, and used for phytohormone measurements, immune complex kinase assays, or histochemical analysis of lignin.

\section{RKN penetration assay.}

In total, 200 and 500 second-stage juvenile RKNs were used for tomato and Arabidopsis, respectively. Inoculation of roots with RKNs was performed in accordance with the procedure described by Fujimoto and associates (2011a). Briefly, roots of plants were inoculated with RKNs by injecting a suspension solution containing RKNs into the sand surrounding the roots

Table 1. List of primers and the corresponding clones used for real-time reverse-transcriptase polymerase chain reaction analysis

\begin{tabular}{|c|c|c|c|}
\hline Sequence definition & AGI number & Forward primer $\left(5^{\prime}-3^{\prime}\right)$ & Reverse primer $\left(5^{\prime}-3^{\prime}\right)$ \\
\hline$B G L 2 ; \beta-1,3$-glucanase 2 & At3g57260 & GCCGACAAGTGGGTTCAAGA & AACCCCCCAACTGAGGGTT \\
\hline$P R 1$; pathogenesis-related genes 1 & At2g14610 & GTTGCAGCCTATGCTCGGAG & CCGCTACCCCAGGCTAAGTT \\
\hline$V S P 2$; vegetative storage protein 2 & At5g24770 & GTTAGGGACCGGAGCATCAA & AACGGTCACTGAGTATGATGGGT \\
\hline$L O X 2 ;$ lipoxygenase 2 & At3g45140 & TTGCTCGCCAGACACTTGC & GGGATCACCATAAACGGCC \\
\hline$P D F 1.2$; plant defensin 1.2 & At5g44420 & CCATCATCACCCTTATCTTCGC & TGTCCCACTTGGCTTCTCG \\
\hline chiB; basic chitinase B & At3g12500 & ACGGAAGAGGACCAATGCAA & GTTGGCAACAAGGTCAGGGT \\
\hline AtPDR 12; ABC transporter $\mathrm{G}$ family member 40 & Atlg15520 & ATGCTTTCGCTCAGGTACTTCA & CTTCACCGCCGTCCACTC \\
\hline PAL1; phenylalanine ammonia-lyase 1 & At $2 \mathrm{~g} 37040$ & TTCСТСТСТССТАСАTCGCC & ACACCATTGACGCCATTCC \\
\hline $\mathrm{C} 4 \mathrm{H}$; trans-cinnamate 4-monooxygenase & At $2 \mathrm{~g} 30490$ & TCCCAGCAGAAAGCAAAATCCTT & GCACATACCTGAAGTCATTACC \\
\hline$C C R 2$; cinnamoyl-CoA reductase & AT1G80820 & GACGATCCCGTAAGTAGACAATT & ACAATGGCTTGAGTGTCACG \\
\hline$C B P 20$; cap-binding protein 20 & At5g44200 & CCTTGTGGCTTTTGTTTCGTC & ACACGAATAGGCCGGTCATC \\
\hline
\end{tabular}


of each plant. After 7 days of inoculation, the roots of plants were washed free of all sand and stained with acid fuchsin, as described previously (McBeth et al. 1941). The total numbers of stained RKNs were counted using a light microscope.

\section{Nematicidal activity assay and infectivity assay.}

For the nematicidal activity assay, approximately $100 \mathrm{RKN}$ in $1 \mathrm{ml}$ of nematode suspension were added to $1 \mathrm{ml}$ of $200 \mu \mathrm{M}$ sclareol solution or control solution ( $0.2 \%$ methanol) in 24-well tissue culture plates and incubated in the dark at $25^{\circ} \mathrm{C}$. At $1,3,6$, 12,24 , and $48 \mathrm{~h}$ after the onset of incubation, the numbers of motile and nonmotile RKNs were counted. During these trials, nonmotile RKNs at the second stage were prodded with $\mathrm{NaOH}$ at $1 \mathrm{~mol} / \mathrm{liter}$ to check for a response, as described by Chen and Dickson (2000).

For infectivity assays, approximately $200 \mathrm{RKNs}$ were soaked with a solution containing $100 \mu \mathrm{M}$ sclareol or $0.1 \%$ methanol in a petri dish. Two days after soaking, RKNs were used for RKN penetration assays using tomato plants.

\section{Phytohormone measurement.}

For the measurement of ET released from Arabidopsis plants, five 10-week-old plants (Col-0) grown in the hydroponic system were placed in a solution containing $100 \mu \mathrm{M}$ sclareol or $0.1 \%$ methanol, with the roots submerged in the solution in a sealed $40-\mathrm{ml}$ glass vial, and incubated in a chamber maintained at $25^{\circ} \mathrm{C}$ with $16 \mathrm{~h}$ of light. After $48 \mathrm{~h}$, a sample was withdrawn from the headspace and analyzed for ET, as described previously (Seo et al. 2007).

Extraction and quantification of SA and JA was performed in accordance with the procedure described by Seo and associates (2007).

\section{RNA extraction and real-time RT-PCR.}

Total RNA ( $3 \mu \mathrm{g})$ extracted from roots using TRIzol reagent (Invitrogen) was reverse transcribed into cDNA using random oligo-hexamers and Superscript III reverse transcription in accordance with the manufacturer's instructions (Invitrogen). Real-time RT-PCR was carried out using SYBR Green PCR Master Mix (Applied Biosystems) using the first-strand cDNA as a template on a sequence detector system (ABI Prism $7900 \mathrm{HT}$; Applied Biosystems). Expression of CBP20 was used for normalization as a standard control gene of Arabidopsis. Nucleotide sequences of the gene-specific primers are described in Table 1. Each treatment had three replicates (five plants per sample).

\section{Protein extraction, immune complex kinase assay, and immunoblotting analysis.}

Roots were harvested at the indicated time intervals, immediately frozen in liquid $\mathrm{N}_{2}$, and stored at $-80^{\circ} \mathrm{C}$ until use. Protein extraction was performed in accordance with the procedure described by Seo and associates (2007). Anti-MPK3 and anti-MPK6 antibodies were generated from rabbits immunized with synthetic peptides corresponding to amino acid residues 359 to 370 (QEAIALNPTYG) for MPK3 and 384 to 395 (REALAFNPEYQQ) for MPK6, and purified using each antigen. The affinity-purified antibodies were used for each analysis. Immune complex kinase assays using $50 \mu \mathrm{g}$ of total protein and immunoblotting analysis using $25 \mu \mathrm{g}$ of total protein were performed as described previously (Seo et al. 2007).

\section{Lignin analysis.}

Harvested roots were used to either quantify thioglycolic acid lignin (Suzuki et al. 2009) or histochemically detect lignin with phloroglucinol (Caño-Delgado et al. 2000). Each treatment had three replicates (five plants per sample).

\section{Statistical analyses.}

The number of RKNs detected in roots in each experiment was adapted to two-way analysis of variance followed by Tukey's test, and Student's $t$ test was used to estimate the influence of such differences (Sokal and Rohlf 1995). The mean of the dependent variables (i.e., number of RKNs in the root or egg masses on the root) was logarithmically transformed to obtain normality and homoscedasticity. These analyses were performed using JMP software (version 10.0.2; SAS Institute Inc., Cary, NC, U.S.A.).

\section{ACKNOWLEDGMENTS}

This study was supported by a Grant-in-Aid for Scientific Research from the Ministry of Education, Culture, Sports, Science and Technology (MEXT) of Japan; the Japanese Program for the Promotion of Basic and Applied Research for Innovation in Bio-oriented Industry (BRAIN), Japan; and a grant from Cross-Ministerial Strategic Innovation Promotion Program, (SIP) of Council for Science, Technology and Innovation Japan. We thank Y. Takeuchi (Graduate School of Agriculture, Kyoto University) for critical reading of the manuscript and Y. Osakabe (RIKEN) for help with lignin measurements.

\section{LITERATURE CITED}

Abe, H., Ohnishi, J., Narusaka, M., Seo, S., Narusaka, Y., Tsuda, S., and Kobayashi, M. 2008. Function of jasmonate in response and tolerance of Arabidopsis to thrip feeding. Plant Cell Physiol. 49:68-80.

Bailey, J. A., Carter, G. A., Burden, R. S., and Wain, R. L. 1975. Control of rust disease by diterpenes from Nicotiana glutinosa. Nature 255: 328-329.

Bhattarai, K. K., Xie, Q. G., Mantelin, S., Bishnoi, U., Girke, T., Navarre, D. A., and Kaloshian, I. 2008. Tomato susceptibility to root-knot nematodes requires an intact jasmonic acid signaling pathway. Mol. Plant-Microbe Interact. 21:1205-1214.

Campbell, E. J., Schenk, P. M., Kazan, K., Penninckx, I. A., Anderson, J. P., Maclean, D. J., Cammue, B. P. A., Ebert, P. R., and Manners, J. M. 2003. Pathogen-responsive expression of a putative ATP-binding cassette transporter gene conferring resistance to the diterpenoid sclareol is regulated by multiple defense signaling pathways in Arabidopsis. Plant Physiol. 133:1272-1284.

Caniard, A., Zerbe, P., Legrand, S., Cohade, A., Valot, N., Magnard, J.-L., Bohlmann, J., and Legendre, L. 2012. Discovery and functional characterization of two diterpene synthases for sclareol biosynthesis in Salvia sclarea (L.) and their relevance for perfume manufacture. BMC Plant Biol. 12:119.

Caño-Delgado, A. I., Metzlaff, K., and Bevan, M. W. 2000. The eli1 mutation reveals a link between cell expansion and secondary cell wall formation in Arabidopsis thaliana. Development 127:3395-3405.

Chen, S. Y., and Dickson, D. W. 2000. A technique for determining live second-stage juveniles of Heterodera glycines. J. Nematol. 32:117121.

Cheng, A.-X., Lou, Y.-G., Mao, Y.-B., Lu S., Wang, L.-J., and Chen, X.-Y. 2007. Plant terpenoids: biosynthesis and ecological functions. J. Integr. Plant Biol. 49:179-186.

Fudali, S. L., Wang, C., and Williamson, V. M. 2013. Ethylene signaling pathway modulates attractiveness of host roots to the root-knot nematode Meloidogyne hapla. Mol. Plant-Microbe Interact. 26:75-86.

Fujimoto, T., Tomitaka, Y., Abe, H., Tsuda, S., Futai, K., and Mizukubo, T. 2011a. Expression profile of jasmonic acid-induced genes and the induced resistance against the root-knot nematode (Meloidogyne incognita) in tomato plants (Solanum lycopersicum) after foliar treatment with methyl jasmonate. J. Plant Physiol. 168:1084-1097.

Fujimoto, T., Tomitaka, Y., Abe, H., Tsuda, S., Futai, K., and Mizukubo, T. 2011b. Jasmonic acid signaling pathway of Arabidopsis thaliana is important for root-knot nematode invasion. Nematol. Res. 41:9-17.

Glazer, I., Apelbaum, A., and Orion, D. 1985. Effect of inhibitors and stimulators of ethylene production on gall development in Meloidogyne javanica-infected tomato roots. J. Nematol. 17:145-149.

Glazer, I., Orion, D., and Apelbaum, A. 1983. Interrelationships between ethylene production, gall formation, and root-knot nematode development in tomato plants infected with Meloidogyne javanica. J. Nematol. 15:539-544.

Grec, S., Vanham, D., de Ribaucourt, J. C., Purnelle, B., and Boutry, M. 2003. Identification of regulatory sequence elements within the 
transcription promoter region of $N p A B C 1$, a gene encoding a plant $\mathrm{ABC}$ transporter induced by diterpenes. Plant J. 35:237-250.

Jasiński, M., Stukkens, Y., Degand, H., Purnelle, B., Marchand-Brynaert, J. and Boutry, M. 2001. A plant plasma membrane ATP binding cassettetype transporter is involved in antifungal terpenoid secretion. Plant Cell 13:1095-1107.

Kazan, K., and Lyons, R. 2014. Intervention of phytohormone pathways by pathogen effectors. Plant Cell 26:2285-2309.

Kennedy, B. S., Nielsen, M. T., Severson, R. F., Sisson, V. A., Stephenson, M. K., and Jackson, D. M. 1992. Leaf surface chemicals from Nicotiana affecting germination of Peronospora tabacina (adam) sporangia. J. Chem. Ecol. 18:1467-1479.

Leon-Reyes, A., Spoel, S. H., De Lange, E. S., Abe, H., Kobayashi, M., Tsuda, S., Millenaar, F. F., Welschen, R. A. M., Ritsema, T., and Pieterse, C. M. 2009. Ethylene modulates the role of NONEXPRESSOR OF PATHOGENESIS-RELATED GENES1 in cross talk between salicylate and jasmonate signaling. Plant Physiol. 149:1797-1809.

Li, G., Meng, X., Wang, R., Mao, G., Han, L., Liu, Y., and Zhang, S. 2012. Dual-level regulation of ACC synthase activity by MPK3/MPK6 cascade and its downstream WRKY transcription factor during ethylene induction in Arabidopsis. PLoS Genet. 8:e1002767.

McBeth, C. W., Taylor, A. L., and Smith, A. L. 1941. Note on staining nematodes in root tissues. Pages 3-6 in: Proc. Helminthol. Soc. Wash. 16.

Milligan, S. B., Bodeau, J., Yaghoobi, J., Kaloshian, I., Zabel, P., and Williamson, V. M. 1998. The root knot nematode resistance gene Mi from tomato is a member of the leucine zipper, nucleotide binding, leucine-rich repeat family of plant genes. Plant Cell 10:1307-1319.

Nombela, G., Williamson, V. M., and Muñiz, M. 2003. The root-knot nematode resistance gene Mi-1.2 of tomato is responsible for resistance against the whitefly Bemisia tabaci. Mol. Plant-Microbe Interact. 16:645-649.

Pedras, M. S. C., Okanga, F. I., Zaharia, I. L., and Khan, A. Q. 2000. Phytoalexins from crucifers: synthesis, biosynthesis, and biotransformation. Phytochemistry 53:161-176.

Raes, J., Rohde, A., Christensen, J. H., Van de Peer, Y., and Boerjan, W. 2003. Genome-wide characterization of the lignification toolbox in Arabidopsis. Plant Physiol. 133:1051-1071.

Robert-Seilaniantz, A., Grant, M., and Jones, J. D. 2011. Hormone crosstalk in plant disease and defense: more than just jasmonate-salicylate antagonism. Annu. Rev. Phytopathol. 49:317-343.

Rodriguez, M. C., Petersen, M., and Mundy, J. 2010. Mitogen-activated protein kinase signaling in plants. Annu. Rev. Plant Biol. 61:621-649.

Schmelz, E. A., Alborn, H. T., Banchio, E., and Tumlinson, J. H. 2003. Quantitative relationships between induced jasmonic acid levels and volatile emission in Zea mays during Spodoptera exigua herbivory. Planta 216:665-673.
Seo, S., Katou, S., Seto, H., Gomi, K., and Ohashi, Y. 2007. The mitogenactivated protein kinases WIPK and SIPK regulate the levels of jasmonic and salicylic acids in wounded tobacco plants. Plant J. 49:899-909.

Seo, S., Gomi, K., Kaku, H., Abe, H., Seto, H., Nakatsu, S., Neya, M., Kobayashi, M., Nakaho, K., Ichinose, Y., Mitsuhara, I., and Ohashi, Y. 2012. Identification of natural diterpenes that inhibit bacterial wilt disease in tobacco, tomato and Arabidopsis. Plant Cell Physiol. 53:1432-1444.

Sokal, R. R., and Rohlf, F. J. 1995. Biometry, 3rd Ed. W.H. Freedman \& Co., New York.

Suzuki, S., Suzuki, Y., Yamamoto, N., Hattori, T., Sakamoto, M., and Umezawa, T. 2009. High-throughput determination of thioglycolic acid lignin from rice. Plant Biotechnol. 26:337-340.

Uno, Y., Furihata, T., Abe, H., Yoshida, R., Shinozaki, K., and YamaguchiShinozaki, K. 2000. Arabidopsis basic leucine zipper transcription factors involved in an abscisic acid-dependent signal transduction pathway under drought and high-salinity conditions. Proc. Natl. Acad. Sci. U.S.A. 97:11632-11637.

Vogt, T. 2010. Phenylpropanoid biosynthesis. Mol. Plant 3:2-20.

Volkmar, K. M. 1991. Abscisic acid and ethylene increase in Heterodera avenae-infected tolerant or intolerant oat cultivars. J. Nematol. 23: 425-431.

Wang, K. L.-C., Li, H., and Ecker, J. R. 2002. Ethylene biosynthesis and signaling networks. Plant Cell 14 (Suppl. 1):S131-S151.

Wang, C., Lower, S., and Williamson, V. M. 2009. Application of pluronic gel to the study of root-knot nematode behavior. Nematology 11:453-464.

Wuyts, N., Lognay, G., Swennen, R., and De Waele, D. 2006. Nematode infection and reproduction in transgenic and mutant Arabidopsis and tobacco with an altered phenylpropanoid metabolism. J. Exp. Bot. 57: 2825-2835.

Wyss, U., Grundler, F. M. W., and Munch, A. 1992. The parasitic behavior of second stage juveniles of Meloidogyne incognita in root of Arabidopsis thaliana. Nematologica. 38:98-111.

Xie, D. X., Feys, B. F., James, S., Nieto-Rostro, M., and Turner, J. G. 1998 COI1: an Arabidopsis gene required for jasmonate-regulated defense and fertility. Science 280:1091-1094.

Ziegler, J., and Facchini, P. J. 2008. Alkaloid biosynthesis: metabolism and trafficking. Annu. Rev. Plant Biol. 59:735-769.

\section{AUTHOR-RECOMMENDED INTERNET RESOURCES}

RIKEN BioResource Center: www.brc.riken.jp/lab/epd/Eng/catalog/transp.shtml

National Institute of Agrobiological Sciences of Japan: www.gene.affrc.go.jp/about_en.php 